Thomas Oppermann

\title{
Adam von Trott: ein Vorbild für die Demokratie im
} 21. Jahrhundert

Widerstand Demokratie Internationalität 

Thomas Oppermann Adam von Trott: Ein Vorbild für die Demokratie im 21. Jahrhundert Dieses Werk ist lizenziert unter einer

Creative Commons Namensnennung - Weitergabe unter gleichen Bedingungen

$\underline{4.0 \text { International Lizenz. }}$.

(c) $\underset{\mathrm{BY}}{(i)}$ 
erschienen als Heft 2 in der Reihe „Widerstand - Demokratie - Internationalität“ im Universitätsverlag Göttingen 2019 
Thomas Oppermann

\section{Adam von Trott: \\ Ein Vorbild für die Demokratie im \\ 21. Jahrhundert}

Widerstand

Demokratie

Internationalität

Heft 2

Universitätsverlag

Göttingen

2019 
Bibliographische Information der Deutschen Nationalbibliothek

Die Deutsche Nationalbibliothek verzeichnet diese Publikation in der Deutschen Nationalbibliographie; detaillierte bibliographische Daten sind im Internet über $<$ http://dnb.dnb.de $>$ abrufbar.

Gefördert von:

Die Beauftragte der Bundesregierung

für Kultur und Medien

Herausgeber der Reibe

Georg-August-Universität Göttingen

Wilhelmsplatz 1

37073 Göttingen

Stiftung Adam von Trott, Imshausen e.V.

Im Trottenpark 1

36179 Bebra-Imshausen

Dieses Buch ist auch als freie Onlineversion über die Homepage des Verlags sowie über den Göttinger Universitätskatalog (GUK) bei der Niedersächsischen Staats- und Universitätsbibliothek Göttingen (https://www.sub.uni-goettingen.de) erreichbar. Es gelten die Lizenzbestimmungen der Onlineversion.

Layout und Covergestaltung: mediendesign | aronjungermann

(C) 2019 Universitätsverlag Göttingen https:/ / univerlag.uni-goettingen.de ISBN: 978-3-86395-428-4

DOI: https://doi.org/10.17875/gup2019-1226

eISSN: $2698-8828$ 


\section{Vorwort zur Reihe}

Adam von Trott zu Solz (1909-1944) ist erst 35 Jahre alt, als er am 26. August 1944 in Berlin-Plötzensee hingerichtet wird - wenige Wochen nach dem missglückten Attentat auf Hitler am 20. Juli. Der Staat ,ist zur Willkür geworden“ hatte er bereits als junger Jurastudent kurz nach der Machtergreifung der Nationalsozialisten 1933 an seinen Vater geschrieben.

Adam von Trott war von Beginn an Gegner des nationalsozialistischen Regimes und setzte sich seit 1939 aktiv für dessen Sturz ein. Darüber hinaus entwickelte er weitreichende Ideen für ein freies, gemeinsames Europa der Zukunft. Er war ein liberal-demokratisch denkender und handelnder Mensch, gehörte zum Kern der Widerstandsgruppe Kreisauer Kreis und war in enger Zusammenarbeit mit Claus Schenk Graf von Stauffenberg an der Verschwörung vom 20. Juli 1944 beteiligt.

Zum Gedenken an den Göttinger Alumnus und Widerstandskämpfer haben die Universität Göttingen und die Stiftung Adam von Trott, Imshausen e.V. im Jahr 2018 das Projekt „Widerstand - Demokratie - Internationalität" ins Leben gerufen. Gemeinsam organisieren sie Veranstaltungen und initiieren Projekte, die die Auseinandersetzung mit dem historischen Erbe Adam von Trotts für die aktive Gestaltung von Gegenwart und Zukunft nutzbar machen. Dazu gehören öffentliche Vorträge, Tagungen und Konferenzen, Projektseminare, Stipendienprogramme sowie Bildungsangebote für Schülerinnen und Schüler.

Ein herzlicher Dank gilt in diesem Zusammenhang den Bundestagsabgeordneten Thomas Oppermann und Michael Roth sowie den Göttinger Professoren Andreas Busch und Arnulf Quadt, die sich in besonderem Maße für das Kooperationsprojekt eingesetzt haben.

Die vorliegende Schriftenreihe vereint Texte, Vorträge und Reden, die seit 2014 an der Universität und der Stiftung, insbesondere im Zuge von Veranstaltungen in Gedenken an Adam von Trott, entstanden sind. Gemein ist allen Texten ein - teils unmittelbarer, teils mittelbarer - Bezug zur Person Adam von 
Trott und zu den Themen des Kooperationsprojekts: Widerstand und Verantwortung, Demokratie und Frieden, Internationalität und Europa. In einigen Beiträgen steht Adam von Trott selbst im Mittelpunkt der Ausführungen. In anderen Beiträgen, wie beispielsweise in den seit 2016 jährlich stattfindenden Adam-von-Trott-Lectures, stellen die Projektthemen die Brücke zum Leben und Wirken Adam von Trotts her.

Ziel der Reihe ist es, besonders wichtige Beiträge aus der gemeinsamen Arbeit zu dokumentieren und einer interessierten Öffentlichkeit dauerhaft zugänglich zu machen. In diesem Sinne sind alle Texte neben der gedruckten Version auch auf der Seite des Universitätsverlags Göttingen online frei verfügbar.

Wir wünschen Ihnen eine anregende Lektüre!

Prof. Dr. Ulrike Beisiegel

Präsidentin der Georg-August-Universität Göttingen (2011-2019)

Dorothee Engelhard

Vorstandsvorsitzende der Stiftung Adam von Trott, Imshausen e.V.

Weitere Informationen zu der Kooperation zwischen der Universität Göttingen und der Stiftung Adam von Trott sind unter www.uni-goettingen.de/trott zu finden. 


\title{
Adam von Trott: ein Vorbild für die Demokratie im 21. Jahrhundert
}

\author{
von Thomas Oppermann*
}

* In einzelnen Punkten leicht erweiterte schriftliche Fassung meiner Gedenkrede in der Paulinerkirche in Göttingen am 20. Juli 2014 anlässlich des 70. Jahrestags des gescheiterten Umsturzversuchs gegen das NS-Regime am 20. Juli 1944. 


\section{Einleitung}

Heute, am 20. Juli 2014, erinnern wir an den Tag vor 70 Jahren, an dem das Attentat auf Adolf Hitler gescheitert ist. Ich möchte aus der Sicht eines Bundestagsabgeordneten darüber nachdenken, was dieser Gedenktag für uns bedeutet - und ich werde dies am Beispiel des Göttinger Alumnus Adam von Trott tun, der im Mittelpunkt unserer heutigen Veranstaltung steht.

Wie kaum einer seiner Zeitgenossen ist Adam von Trott zu Solz ein politisches Vorbild für die Demokratie im 21. Jahrhundert. Er war eine eindrucksvolle Persönlichkeit, die auch in schwierigen Situationen strikt einer humanistischen Ethik verpflichtet war und gegen den Zeitgeist zu ihren Überzeugungen stand.

\section{Adam von Trott als Europäer und Weltbürger}

Wer sich mit Adam von Trott beschäftigt, dem fällt zuerst seine ausgeprägte Neugier auf fremde Kulturen auf. Das ist keinesfalls selbstverständlich zu einer Zeit, als das gesellschaftliche Klima stark von der nationalsozialistischen Rassenideologie geprägt war.

Sein Freund und Vertrauter Johannes Winckelmann hat es sehr schön auf den Punkt gebracht: von Trott habe sich immer ,zwischen Vaterland und Weltbürgertum, zwischen West und Ost, zwischen Bewahren und Neugestalten “ bewegt: „Jeder neue Standpunkt, jede neue Tatsache, die er in Erfahrung brachte, veranlasste ihn sofort, sein Gedankenbild und seine Vorstellungswelt kritisch zu erweitern oder in ergänzendem Sinne auszubilden." Auch angesichts der bitteren Zeitläufte habe von Trott sich nicht mit Wehklagen aufgehalten, sondern versucht, konstruktiv zu denken. Es waren, sagte Winckelmann, „mundiale Perspektiven einer Welt im Umbruch, einer Welt von morgen", die ihn interessierten. Ihm war es zeitlebens wichtig, die Welt kennen zu lernen. Insbesondere zur NS-Zeit war es alles andere als üblich, nicht nur durch ganz Europa und in die USA, sondern sogar bis nach China zu reisen. Von allen seinen Studienreisen, die er als bri- 
tischer Stipendiat unternehmen konnte, brachte er neue Erfahrungen und neues Wissen mit. Das machte ihn zu einem echten Weltbürger. „Diese weiten Welten“, schrieb er Anfang 1940 auf dem Pazifik einer Freundin nach London, „haben mich mit mehr Freude, Unabhängigkeit und Widerstandskraft ausgerüstet, als ich je hätte hoffen können. “Im gleichen Brief sieht er ein „Weltzeitalter“ voraus, das ganz neue geistige Anstrengungen erfordern werde. Wir nennen das heute Globalisierung.

Seine Weltbürgerschaft hat ganz sicher dazu beigetragen, ihn gegen jede Form von Nationalismus zu immunisieren. Mit weitreichenden Konsequenzen: Als Adolf Hitler an die Macht kam und Deutschland schon lange im Zeichen eines übersteigerten Nationalismus stand, nahm Adam von Trott bereits eine entschieden transnationale und europäische Perspektive ein.

Als begeisterter Anhänger des Europagedankens konzipierte er 1939 in New York die Ideen einer europäischen Zollund Währungsunion, eines gemeinsamen europäischen Gerichtshofes und eines gesamteuropäischen staatsbürgerlichen Status, der die Grundlage zu weiteren Zusammenlegungen administrativer Souveränität für Teilbereiche des Lebens ermöglichen sollte.

Diese Gedanken waren damals visionär und ihrer Zeit weit voraus. Sein Ziel war ein Deutschland, wie es in der Präambel des Grundgesetzes später formuliert werden sollte, nämlich ,von dem Willen beseelt, als gleichberechtigtes Glied in einem vereinten Europa dem Frieden in der Welt zu dienen." Adam von Trott hatte in seinem Denken und Handeln eine der geglücktesten Formulierungen des Grundgesetzes gleichsam antizipiert.

\section{Adam von Trott als entschiedener Gegner des Nationalsozialismus}

Es ist deshalb nur konsequent, dass Adam von Trott sich bereits 1933 entschieden gegen Hitler aussprach. Zu einer Zeit, als viele die Gefahr der heraufziehenden Terrorherrschaft 
noch unterschätzten. Zeitlebens hegte er auch nicht die geringsten Sympathien für den Nationalsozialismus, geschweige denn den Antisemitismus oder eine andere Spielart des Rassismus. Adam von Trott war immer ein überzeugter Anhänger der Werte, die das Fundament unserer Demokratie bilden.

Seinen Abschiedsbrief aus der Todeszelle an seine Frau nutzte er zu einer finalen, aber unter den gebotenen Umständen höchst subtilen Abrechnung mit Hitler und dem NS-Regime. Die wenigen Zeilen, die der verurteilte Landesverräter am 26. August $1944 \mathrm{im}$ Gefängnis Berlin-Plötzensee schrieb, lesen sich auf den ersten Blick recht unverfänglich: „Ich habe in den letzten Tagen noch das Purgatorio gelesen, auch Maria Stuart und, was mich seltsam stark berührte, den Jürg Jenatsch. Sonst hatte ich solches wenig - aber sehr vieles in mir, was ich in Ruhe bewegen und klarlegen konnte. So sei um mich nicht zu bekümmert - alles ist ja im Grunde klar, wenn auch tief schmerzlich.“

Er spricht damit von drei Büchern, die er in der Todeszelle gelesen hatte: Den Abschnitt Purgatorio (Fegefeuer) aus Dantes Divina Comedia, der Göttlichen Komödie, Schillers Drama Maria Stuart und Conrad Ferdinand Meyers Roman Jürg Jenatsch.

Sollte es ein Zufall sein, dass er diese drei Bücher hier erwähnt? In einem Abschiedsbrief aus der Todeszelle, in einem Dokument also, in dem der Schreibende wie alle späteren Leser selbstverständlich jedes einzelne Wort sehr genau zu bedenken haben? Das glaube ich nicht.

Was er in den Jahren seiner Tätigkeit im Widerstand perfekt gelernt hatte, war das subversive Schreiben und Sprechen: Das war eine der wichtigsten Strategien, um zu überleben. Deshalb ist es legitim, auch in diesem Abschiedsbrief mehr zu vermuten, als auf den ersten Blick hin dort zu lesen steht.

Warum wählte er ausgerechnet diese drei Bücher aus zwei davon Klassiker der Weltliteratur, dazu ein heute fast vergessener historischer Roman? Alle drei Werke haben gemeinsam, dass sie sich mit Willkürherrschaft und dem Widerstand dagegen befassen.

Im Purgatorio schildert Dante den furchtbaren Höllenpfuhl, in dem die Mörder im kochenden Blutstrom gepeinigt werden - in dessen furchtbarsten Tiefen die Tyrannen, Er- 
oberer und Gewaltherrscher schmoren: ganz am Grunde Attila der Hunne, der Schlächter, den man im Widerstand und im Ausland oft in einem Atemzug mit Adolf Hitler genannt hat, wie es zum Beispiel das britische Magazin The Spectator am 14. April 1939 tat, in der ein anonymer deutscher Korrespondent Hitler den „neuen Attila [nannte], eine Geißel Gottes, [...] die Inkarnation des Deutschtums, die Personifizierung des Furor Teutonicus.“

Friedrich Schillers Maria Stuart wird als „Mordanstifterin [...] zum Tode verurteilt wegen Hochverrat" an Königin Elisabeth, obwohl sie unschuldig ist. Und die zum Tode verurteilte Maria spricht unmittelbar vor ihrer Hinrichtung die Worte: „Mir wird ein Glück zuteil, wie ich es nimmer gehoffet, dass mein Nachruhm doch nicht ganz in meiner Feinde Händen ist." Das ist eine Hoffnung, die auch Adam von Trott gehegt haben dürfte.

Besonders hervorgehoben in der kurzen Liste ist aber Meyers historischer Roman Jürg Jenatsch, der in den Wirren des Dreißigjährigen Krieges spielt und der Adam von Trott so „seltsam stark“ berührt hat.

Jürg Jenatsch, die Hauptfigur des dritten Buches, das Adam von Trott erwähnt, ist ein Nationalist, der fanatisch und gewissenlos für seine Heimat Bünden kämpft, um dabei furchtbare Schuld auf sich zu laden, unter anderem den Mord an seinem persönlichen und privaten Gegenspieler, dem Feldherrn Pompejus Planta. Doch schließlich wird auch Jenatsch selbst Opfer eines Attentats, und ganz am Ende des Romans werden seine Mörder als „die Werkzeuge eines notwendigen Schicksals" bezeichnet, die man nicht vor Gericht stellen wird.

Sollte die Vermutung stimmen, dass in von Trotts Hinweisen auf seine letzten Lektüren vor der Hinrichtung auch ein Subtext verborgen ist, der die historische Notwendigkeit des Attentats auf Adolf Hitler, auf die Geißel Gottes rechtfertigt, die in die tiefste Hölle gehört, dann würde dieser gut zu dem Notizbucheintrag passen, den Adam von Trott als 26-Jähriger im Jahre 1935 schrieb: „,Wenn wir uns schon mit einer Epoche abfinden müssen, in der die größere Wahrscheinlichkeit für ein vorzeitiges Lebensende steht, sollten wir doch wenigstens dafür sorgen, dass es Sinn hat zu sterben - gelebt zu haben.“ 
Diesen Worten verdankt die große Adam von Trott zu SolzBiographie von Benigna von Krusenstjern, die im Wallstein Verlag erschienen ist, ihren Titel, und das, was man vielleicht als ein Lebensmotto verstehen kann, würde dann ganz am Ende des Lebens noch einmal unterstrichen durch die Verweise auf Dante, Schiller und Conrad Ferdinand Meyer: Die moralische Gewissheit, richtig gehandelt zu haben und die Hoffnung darauf, dass es eine Zeit nach Hitler geben könnte, welche die Verschwörung gegen den Terrorstaat als Recht, und nicht mehr als Verrat beurteilen würde.

Auch diesen Gedanken von Trotts hat später das Grundgesetz in Artikel 20 IV aufgegriffen. Dort heißt es: „Gegen jeden, der es unternimmt, diese Ordnung zu beseitigen, haben alle Deutschen das Recht zum Widerstand, wenn andere Abhilfe nicht möglich ist." Das ist ganz genau dasjenige Recht, für das Adam von Trott bis zu seinem Tode mit ganzer Kraft und ohne Rücksicht auf die möglichen Konsequenzen eingetreten ist.

Er ist 26 Jahre alt gewesen, als er 1935 eine Auswahl der Schriften Heinrich von Kleists herausgab. Mit Blick auf den Rebellen Michael Koblhaas legte er auch im Vorwort dieses Buches grundlegende Gedanken über das Recht auf Widerstand nieder. Als „Kern aller politischen Existenz“ bezeichnete er hier die „Möglichkeit der freien Gewissensentscheidung“ gegen eine Politik, die die echte menschliche „Ordnung zu gefährden vermag“: „Je unsicherer es mit der Welt überhaupt bestellt ist, desto sicherer ist es notwendig, für dieses Recht zu kämpfen."

Auch das ist ein Beispiel für jenes subversive Sprechen beziehungsweise Schreiben, das im Widerstand gegen den Nationalsozialismus notwendig geworden war: Was er vorgeblich über Heinrich von Kleists Michael Kohlhaas sagte, war auf den Nationalsozialismus gemünzt. Das Recht zu kämpfen - das sah Adam von Trott ab 1933 als seine Pflicht an. Und dieser Pflicht blieb er stets treu. 


\section{Adam von Trotts Position in der Gruppe der Widerstandskämpfer vom 20. Juli}

„Frieden schaffen ohne Waffen“ - daran war in den Jahren nach 1933 nicht zu denken. Die Terrorherrschaft der Nazis konnte nicht ohne Waffen beseitigt werden. Die Widerstandsgruppe um Stauffenberg sah ein mörderisches Attentat gegen Hitler als einzige Möglichkeit, erfolgreich für Frieden und Freiheit eintreten zu können - ein schwerer Gewissenskonflikt, der auch den nachdenklichen Humanisten Adam von Trott bewegt hat.

Die Gruppe der Verschwörer war sehr heterogen. Da waren auf der einen Seite die aristokratisch und nationalkonservativ gesonnenen, elitär denkenden Protagonisten wie von Stauffenberg selbst, auf der anderen Seite standen Christen und Theologen wie Dietrich Bonhoeffer und Sozialdemokraten wie Julius Leber - oder eben Menschen wie Adam von Trott, der sich bereits seit den 20er Jahren immer wieder zur Demokratie bekannte. Und ich bin stolz darauf, dass es eben die Sozialdemokratie war, die auch sein politisches Denken, wie dasjenige Julius Lebers, bestimmt hat. In einem seiner kleinen Notizbücher hat Trott den Grundsatz festgehalten: „Im Grunde ist jeder ehrliche Humanist auch Sozialist.“

Die ehemalige SPD-Reichstagsabgeordnete Toni Sender, die 1933 aus Deutschland geflohen war, würdigte von Trott 1945 in einem Leserbrief an die New York Times als einen „Vorkämpfer hoher demokratischer Ideale“, und ein solcher ist er tatsächlich immer gewesen. Er war es ja auch, der Willy Brandt in seinem Stockholmer Exil ins Vertrauen über die Attentatspläne zog und ihn fragte, ob er einer zivilen Regierung nach Adolf Hitler zur Verfügung stehen würde.

In der Gruppe um Stauffenberg war er maßgeblich für internationale Kontakte zu den Kriegsgegnern zuständig. Er war dafür aus mehreren Gründen prädestiniert: wegen seiner fließenden Englisch-Kenntnisse, seiner kosmopolitischen Haltung, seiner beruflichen Position in der Informationsabteilung des Auswärtigen Amtes und seiner vielfältigen ausländischen Beziehungen. 


\section{Schluss-Würdigung}

Adam von Trott war ein Vorkämpfer und Vordenker demokratischer Ideale für ein friedliches, freies Deutschland nach Ende der NS-Terrorherrschaft.

Das, was Adam von Trott dachte, was er tat und was er schrieb, ist aus politischer Sicht heute aufsehenerregend modern. Er war Patriot, aber kein Nationalist. Rassismus und nationalstaatliche Hegemonie waren ihm zutiefst wesensfremd. Er dachte global, entschied sich für eine international geprägte Bildung. Er glaubte an die Existenz einer übernationalen Wertegemeinschaft in Europa und war ein Vordenker der europäischen Integration. Er forderte eine Demokratie, die ,in weiteren Kanälen“" zur Geltung komme als durch Parlamente. Er scheute sich nicht, zur Erreichung solcher Ziele immer wieder sein Leben für den Sturz des NS-Regimes einzusetzen.

Als sich von Trott von Oktober bis Dezember 1939 in den USA aufhielt, überwachte das FBI ihn als mutmaßlichen Spion der Nazis. Bei einem Dinner im hoch angesehenen Yale Club hielt er eine Rede, in der er offen darüber sprach, dass der Gestapo-Terror in Deutschland das Volk in so große Angst versetzt habe, dass es keinesfalls zu einem zivilen Aufstand gegen das Hitler-Regime kommen würde - ein Umsturz könne einzig durch oppositionelle Generäle vollzogen werden. Diesen aber müsse man garantieren, dass Deutschland nach einem Friedensschluss als unabhängiger Staat in den Grenzen von 1933 erhalten bleiben würde. Das Militär dürfte die Regierung dabei nur übergangsweise übernehmen, damit eine zivile Regierung rasch einen Staat des Rechtes in Deutschland schaffen könne.

Seine Beurteilung der Situation des Widerstands in Deutschland wollte er übrigens auch Präsident Roosevelt vortragen - aber dazu kam es leider nicht. Adam von Trott erreichte nur eine Einladung zu einer Teegesellschaft bei der Präsidentengattin Eleanor Roosevelt, die ihn dabei angeblich mit folgenden Worten eingeführt haben soll: „This is Adam von Trott, a friend of ours, who will tell you all about the German underground movement."

Aufgrund solcher Auftritte nannten Freunde von Trotts Verhalten im Ausland „haarsträubend unvorsichtig“. Damit 
hatten sie gewiss Recht. Wäre ein Spion der Gestapo im Raum gewesen, hätte das von Trotts Todesurteil bedeutet. Aber seine Zivilcourage war offenkundig größer als seine Angst vor Verrat.

Noch in der Todeszelle blieb es ungewöhnlich, was Adam von Trott dachte und was er schrieb. Im Abschiedsbrief an seine Frau schrieb der verurteilte Landesverräter am 26. August 1944: „Am meisten schmerzt mich, unserm Land die besonderen Kräfte und Erfahrungen, die ich in fast zu einseitiger Konzentration auf seine außenpolitische Behauptung unter den Mächten in mir ausgebildet hatte, nun vielleicht nie mehr dienend zur Verfügung stellen kann. Hier hätte ich wirklich noch helfen und nützen können. [...] So muss ich hoffen, dass auch ohne mich von vielen dieser Verbindungen auch so Verständnis und Hilfe zufließen wird, wenn es einmal wieder nötig und wünschenswert sein sollte. Aber ein Sämann überlässt nicht gerne knospende Saaten anderen zur weiteren Bearbeitung, denn zwischen Saat und Ernte liegen ja noch so viele Stürme.“

Mit den Stürmen, die von Trott hier ansprach, war gewiss die unweigerliche, kurz bevorstehende Kriegsniederlage Deutschlands gemeint. Er hatte die Hoffnung, dass diejenige Saat, die er mit vielen Schreiben und Gesprächen im Widerstand und unter den Kriegsgegnern Deutschlands ausgebracht hatte, doch noch aufgehen würde.

Dass wir heute mit Gästen aus Oxford hier zusammengekommen sind, um ihn 70 Jahre nach dem Attentat auf Adolf Hitler zu würdigen, zeigt auch, dass sich diese Hoffnungen erfüllt haben. Die politische Saat, an der dem „Sämann“ Adam von Trott noch in seiner Todeszelle so sehr gelegen war, ist schließlich aufgegangen. Heute leben wir in einer Gesellschaft, die stolz darauf sein darf, dass auch während der dunkelsten Stunden unserer Geschichte Menschen wie Adam von Trott unsere Grundwerte verteidigt und dafür ihr Leben aufs Spiel gesetzt haben. Es hätte ihn sehr gefreut, mitzuerleben, dass die Prinzipien von Freiheit, Recht und Demokratie, für die er so leidenschaftlich eintrat, heute fest in unserem Grundgesetz verankert sind. 
Anlässlich des 70. Jahrestags des Umsturzversuchs gegen das NS-Regime am 20. Juli 1944 würdigt Thomas Oppermann den Göttinger Alumnus und Widerstandskämpfer Adam von Trott zu Solz als mutigen Gegner der Nazi-Diktatur und als Vorbild für unsere heutige Demokratie. Aus seiner persönlichen Sicht als Bundestagsabgeordneter hebt Oppermann besonders von Trotts visionäre Gedanken zu einem vereinten, friedlichen Europa hervor. Kurze Streiflichter in die Biografie von Trotts geben einen Einblick in sein bewegtes Leben und zeigen, welch Inspiration und Aktualität im Denken und Wirken des Widerstandskämpfers zu finden sind.

Thomas Oppermann ist Abgeordneter der SPD im Deutschen Bundestag. Er studierte Rechtswissenschaften an der Universität Göttingen und hat sich in besonderem Maße für die Kooperation zwischen der Universität Göttingen und der Stiftung Adam von Trott eingesetzt. 